\title{
Incidence of Sustained Ventricular Tachycardia in Patients with Prolonged QTe After the Administration of Azithromycin: A Retrospective Study
}

\author{
Steven P. Sears ${ }^{1}$ (D) Trevor W. Getz ${ }^{2}$ - Christopher O. Austin ${ }^{3} \cdot$ William C. Palmer $^{4}$ • \\ Evelyn A. Boyd ${ }^{2} \cdot$ Fernando F. Stancampiano $^{1}$
}

Published online: 14 March 2016

(c) The Author(s) 2016. This article is published with open access at Springerlink.com

\begin{abstract}
Background Azithromycin has been associated with abnormalities of cardiac repolarization and development of torsades de pointes. Observational data suggest that the risk of death from cardiovascular causes is increased in patients taking azithromycin. Little is known regarding the risk of ventricular arrhythmia in patients with prolongation of the corrected QT interval who receive azithromycin.

Objective The purpose of this study was to determine the incidence of sustained ventricular tachycardia in patients with prolonged corrected QT (QTc) who subsequently received azithromycin.

Methods We performed a retrospective cohort analysis of the incidence of sustained ventricular tachycardia in patients with prolonged QTc (greater than $450 \mathrm{~ms}$ ) who successively received intravenous (IV) and/or oral azithromycin. Patients hospitalized in a tertiary care teaching hospital between November 2009 and June 2012 were included in the study. The primary outcome was sustained ventricular tachycardia documented in patients on telemetry.
\end{abstract}

Results Of the 103 patients enrolled in the study, only one patient experienced the primary outcome $(0.97 \%)$. The

Steven P. Sears

sears.steven@mayo.edu

1 Department of Internal Medicine, Mayo Clinic, 4500 San Pablo Road S, Jacksonville, FL 32224, USA

2 CRISP Investigative Research Program, Mayo Clinic, Jacksonville, FL, USA

3 Department of Cardiology, Mayo Clinic, Jacksonville, FL, USA

4 Department of Gastroenterology and Hepatology, Mayo Clinic, Jacksonville, FL, USA event occurred 1 day after the administration of a single dose of $500 \mathrm{mg}$ IV azithromycin.

Conclusion The risk of sustained ventricular tachycardia was $0.97 \%$ in our cohort of patients with prolonged QTc who subsequently received azithromycin. Given the small size of this study, additional research is needed to determine the true incidence of arrhythmia in the population.

\section{Key Points}

Azithromycin has been associated with prolongation of the QTc interval and development of torsades de pointes.

Previously, there were no data detailing the incidence of sustained ventricular tachycardia in patients with known prolonged QTc who were subsequently given azithromycin.

In a small cohort of patients admitted to a tertiary referral medical center, the risk of sustained ventricular tachycardia was less than $1 \%$ in patients with prolonged QTc who subsequently received azithromycin.

\section{Introduction}

Macrolides are commonly used and effective antibiotics for conditions such as respiratory infections and certain sexually transmitted diseases. Early generation macrolides such as erythromycin were found to prolong cardiac repolarization and were associated with torsades de pointes (TdP), a potentially fatal ventricular arrhythmia [1]. Newer 
macrolides were developed for the treatment of atypical organisms in respiratory tract infections. Post-marketing surveillance demonstrated an improved safety profile compared to erythromycin and clarithromycin [2]. Further, azithromycin may provide a survival benefit in older adults with pneumonia when compared with fluoroquinolones [3]. However, as use of the drug became more prevalent, case reports emerged linking azithromycin to prolongation of the QT interval, TdP, and polymorphic ventricular tachycardia. The US Food and Drug Administration's (FDA's) Adverse Event Reporting System has documented over 20 cases of TdP in patients taking azithromycin [4].

A large retrospective cohort study in 2012 showed the risk of both cardiovascular and all-cause mortality in patients who received azithromycin was increased when compared with patients who took amoxicillin [5]. Despite some limitations, this study prompted further investigation, and in 2013 the FDA revised product labels to advise against using azithromycin in patients with prolonged QTc, risk factors for prolonged QTc, and in persons who concomitantly take medications that delay cardiac repolarization. However, no data are available regarding the incidence of ventricular arrhythmias in such populations when they receive azithromycin. In this retrospective study, we evaluated the incidence of sustained ventricular tachycardia in hospitalized patients with prolonged corrected QT interval (QTc), who subsequently received azithromycin (Fig. 1).

\section{Methods}

\subsection{Study Cohort}

Between November 2009 and June 2012, 266 patients at Mayo Clinic in Florida with prolonged QTc greater than $450 \mathrm{~ms}$ (ms) who subsequently received azithromycin were evaluated for inclusion in this retrospective study [6]. Data were collected by retrospective chart review performed by interrogation of our electronic medical record for patients with prolonged QTc, cross-referenced by administration of azithromycin. The resultant database was subsequently manually reviewed and validated by the study authors. Patients were excluded if they were younger than 18 years of age, had a history of sustained ventricular tachycardia (defined as ventricular tachycardia for greater than $30 \mathrm{~s}$ or requiring termination in less than $30 \mathrm{~s}$ due to hemodynamic compromise), had an implanted defibrillator, were using antiarrhythmic medications, or had received azithromycin or another antibiotic within $72 \mathrm{~h}$ prior to admission. The study protocol was approved by the Mayo Clinic Institutional Review Board IRB\# 14-005155. The primary outcome was sustained ventricular tachycardia. Secondary outcomes included cardiac death, all-cause mortality, and length of hospital stay. Baseline characteristics and comorbidities were collected along with concomitant use of QTc prolonging medications. All EKGs were read automatically and reviewed by a staff cardiologist. Bazett's formula was used to correct the QT interval.

\subsection{Statistical Analysis}

Continuous variables were summarized using the sample median. Categorical variables were summarized with number and percentage. The proportion of patients who experienced sustained ventricular tachycardia following the start of azithromycin was estimated along with a $95 \%$ confidence interval (CI). Statistical analysis was performed using JMP (version 9.01; SAS Institute, Inc., Cary, NC, USA).

\section{Results}

A total of 103 patients were included in the final analysis. Subjects may have been excluded for more than one reason. Five patients were excluded due to a history of ventricular tachycardia, 16 due to prior AICD placement, 16
Fig. 1 a Rhythm strip of torsades de pointes in a 91-yearold male with prolonged QTc. b Ventricular tachycardia documented $27 \mathrm{~h}$ following a $500 \mathrm{mg}$ IV dose of azithromycin

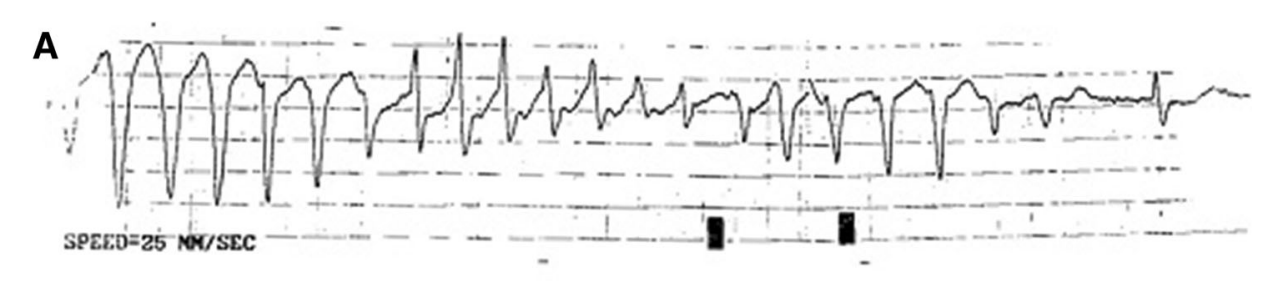

B

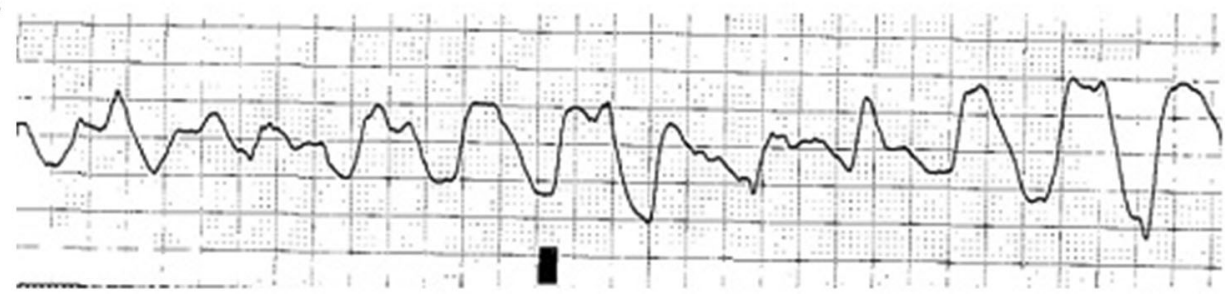


due to concomitant treatment with an antiarrhythmic, 102 excluded for treatment with another antibiotic within $72 \mathrm{~h}$ of admission, 17 were treated with azithromycin prior to admission, and 16 developed prolonged QTc only after a dose of azithromycin. Information on mean age, gender, race, diagnosis at admission, and co-morbid conditions is represented in Table 1. The most common reasons for admission were pulmonary (60\%), cardiovascular (19\%), and renal failure $(7 \%)$. A history of hypertension was noted in $62 \%$ of patients, coronary artery disease in $45 \%$, diabetes mellitus in $31 \%$, and heart failure with reduced ejection fraction in $14 \%$ of patients.

The use of beta-blockers $(50 \%)$ and antidepressants was common $(36 \%)$, whereas antifungal $(2 \%)$ and antipsychotic $(6 \%)$ use was not (Table 2). Indications for azithromycin often differed from initial admission diagnosis and included sexually transmitted infection $(0.9 \%)$, bronchitis $(6.7 \%)$, respiratory failure $(9.7 \%)$, chronic obstructive pulmonary disease exacerbation $(16.5 \%)$, and pneumonia $(66.0 \%)$. The initial dose of azithromycin was $250 \mathrm{mg}$ in nine patients $(9 \%), 500 \mathrm{mg}$ in 92 patients $(89 \%), 1,000 \mathrm{mg}$ in one patient $(0.9 \%)$, and $1,200 \mathrm{mg}$ in one patient $(0.9 \%)$. Intravenous (IV) administration of azithromycin (41\%) was preferred to oral dosing (38\%), and 22 patients received the medication by both routes (22\%). The median duration of treatment was 2 days, (range 1-36 days). The median total dose of azithromycin was $1000 \mathrm{mg}$ (range $250-9000 \mathrm{mg}$ ). The median duration of the QTc interval at time of admission was $474 \mathrm{~ms}$ (range 423-583 ms), while the longest median QTc was $485 \mathrm{~ms}$ (range 451-583 ms). Ninety-four of the 103 patients were monitored on telemetry (91\%).

Only one patient with prolonged QTc developed sustained ventricular tachycardia following administration of azithromycin $(0.97 \%)$, and subsequently expired from the arrhythmia. This patient was critically ill with multiple comorbidities including atrial fibrillation, dementia, severe aortic stenosis, and NYHA class IV congestive heart failure with systolic ejection fraction of $47 \%$. Prior to admission, he took no medications known to prolong the QTc interval but regularly consumed tea containing Zhi Gan Cao, or licorice root. This Chinese herb has been associated with cases of hypokalemia and even ventricular tachycardia and TdP [7]. The primary diagnoses at admission were altered mental status and sepsis, but during the hospitalization, the patient developed stress-related myocardial infarction. On admission the patient's QTc was $486 \mathrm{~ms}$ but later increased to $550 \mathrm{~ms}$. The EKG with QTc interval in excess of $500 \mathrm{~ms}$ was obtained after the patient developed self-terminating TdP. He received one $500 \mathrm{mg}$ IV dose of azithromycin after the index arrhythmia and documentation of prolonged QTc. Recurrent ventricular tachycardia followed by asystolic arrest occurred the following day, $27 \mathrm{~h}$ after the IV
Table 1 Characteristics and co-morbidities of patients with preexisting QT prolongation admitted to a US tertiary-care hospital between November 2009 and June 2012

\begin{tabular}{|c|c|}
\hline Variable & Summary $(N=103)$ \\
\hline Age & $76(26,58,89,98)$ \\
\hline Gender (Male) & $65(63 \%)$ \\
\hline \multicolumn{2}{|l|}{ Race } \\
\hline Caucasian & $93(91 \%)$ \\
\hline African American & $4(4 \%)$ \\
\hline Asian & $3(3 \%)$ \\
\hline Other & $2(2 \%)$ \\
\hline Body mass index & $27(16,23,31,49)$ \\
\hline \multicolumn{2}{|l|}{ Admission diagnosis } \\
\hline Pulmonary & $60(58 \%)$ \\
\hline $\begin{array}{l}\text { Chronic obstructive } \\
\text { pulmonary disease }\end{array}$ & $12(12 \%)$ \\
\hline Pneumonia & $33(32 \%)$ \\
\hline Other & $15(15 \%)$ \\
\hline Cardiovascular & $19(18 \%)$ \\
\hline Congestive heart failure & $12(12 \%)$ \\
\hline Coronary & $4(4 \%)$ \\
\hline Arrhythmia & $3(3 \%)$ \\
\hline Renal failure & $7(7 \%)$ \\
\hline Neurologic & $5(5 \%)$ \\
\hline Vascular & $3(3 \%)$ \\
\hline Non-vascular & $2(2 \%)$ \\
\hline Gastrointestinal & $4(4 \%)$ \\
\hline Liver & $2(2 \%)$ \\
\hline Other & $2(2 \%)$ \\
\hline Infectious & $4(4 \%)$ \\
\hline Oncologic & $3(3 \%)$ \\
\hline Hematologic & $1(1 \%)$ \\
\hline Serum potassium $(\mathrm{mEq} / \mathrm{L})$ & $4.0(2.7,3.6,4.3,6.2)$ \\
\hline Serum calcium (mg/dL) & $8.8(7.4,8.4,9.4,12.5)$ \\
\hline Serum creatinine $(\mathrm{mg} / \mathrm{dL})$ & $1.0(0.4,0.7,1.9,15.4)$ \\
\hline Serum sodium $(\mathrm{mmol} / \mathrm{L})$ & $138(127,135,140,159)$ \\
\hline Glomerular filtration rate & $60(3.4,35,60,60)$ \\
\hline Ejection fraction & $58(22,47,66,73)$ \\
\hline Telemetry & $94(92 \%)$ \\
\hline Diabetes & $32(31 \%)$ \\
\hline Hypertension & $64(62 \%)$ \\
\hline Coronary artery disease & $46(45 \%)$ \\
\hline Congestive heart failure & $14(14 \%)$ \\
\hline
\end{tabular}

The sample median and descriptive statistics (minimum, 25th percentile, 75th percentile, maximum) are given for continuous variables Serum calcium $(N=13)$, and cardiac ejection fraction $(N=43)$

dose of azithromycin (half-life of azithromycin is $68 \mathrm{~h}$ ). Additionally, seven patients died from all-cause mortality $(6.79 \%)$ including one from pneumonia, one from renal failure, one from septic shock, and four from respiratory failure. The median time from first dose of azithromycin to 
Table 2 Medications present on admission along with route and dosing of azithromycin administration with median and maximum duration of the QTc, and length of hospital stay in patients with preexisting QT prolongation admitted to a US tertiary-care hospital between November 2009 and June 2012

\begin{tabular}{ll}
\hline Variable & Summary $(N=103)$ \\
\hline Beta blocker at admission & $52(50 \%)$ \\
Type of beta blocker & \\
$\quad$ Metoprolol & $34(33 \%)$ \\
$\quad$ Carvedilol & $12(12 \%)$ \\
$\quad$ Atenolol & $4(4 \%)$ \\
Other & $2(2 \%)$ \\
Antidepressant at admission & $37(36 \%)$ \\
Type of antidepressant & $16(16 \%)$ \\
$\quad$ Sertraline & $7(7 \%)$ \\
Citalopram & $7(7 \%)$ \\
Paroxetine & $7(7 \%)$ \\
Other & $2(2 \%)$ \\
Antifungal at admission & \\
Type of antifungal & $2(2 \%)$ \\
Fluconazole & $6(6 \%)$ \\
Antipsychotic at admission &
\end{tabular}

The sample median and descriptive statistics (minimum, 25th percentile, 75 th percentile, maximum) are given for continuous variables

the advent of all-cause mortality was 3 days (range 1-37). In all patients who died, the dose of azithromycin was $500 \mathrm{mg}$. Median length of hospitalization for the cohort was 3.2 days (range $0.5-148$ days).

\section{Discussion}

Each year in the USA, between 300,000 and 400,000 people experience sudden cardiac death (SCD) [8], which exceeds the combined mortality of breast cancer, prostate cancer, traffic accidents, firearms, and AIDS [9]. While most cases of SCD are related to underlying structural heart abnormalities or coronary artery disease, a substantial portion is due to arrhythmias $[10,11]$. In the inpatient setting, four of every 1000 patients develop cardiac arrest, of which only $20-50 \%$ are successfully resuscitated [12]. It has been reported that $\mathrm{TdP}$, an insidious polymorphic ventricular arrhythmia, represents $6 \%$ of in-hospital cardiac arrests [13], but the incidence may be higher, as fatal ventricular arrhythmias eventually degrade into pulseless electrical activity or asystole prior to documentation of the instigating dysrhythmia [14].

Torsades de pointes can result from prolongation of the electrocardiographic QTc interval, which represents the period of both cardiac depolarization and repolarization $[8$, $15,16]$. Prolongation of the QTc interval is caused by extension of the myocyte action potential due to increased intracellular current or impairment of phase $3 \mathrm{I}_{\mathrm{k}}$ resulting in accumulation of intracellular potassium [17, 18]. The reduced outward current also imparts a risk for the development of early after-depolarization, in which an extrasystole during the prolonged QT may trigger TdP by re-entry [19]. The length of the QTc interval is dependent upon gender and normally less than $460 \mathrm{~ms}$ in women, and $450 \mathrm{~ms}$ in men [20]. Prolongation of the QTc interval beyond $500 \mathrm{~ms}$ substantially increases the risk of TdP. QTc prolongation may occur as a familial variant of long QT syndrome, but more is more often acquired [21].

Commonly cited risk factors for acquired QTc prolongation include female gender, bradycardia, advanced age, electrolyte disturbances such as hypokalemia and hypomagnesemia, heart failure, and conditions that reduce the hepatic metabolism of medications [17, 19]. Medicationinduced QTc prolongation is more common, with a myriad of agents having been implicated [21]. The risk of medication-induced TdP may be additive with polypharmacy, as the mechanism of QTc prolongation differs between drug classes [17]. As the population ages, polypharmacy is more abundant and hospitalizations become more frequent. In prior studies, $25 \%$ of patients admitted to the hospital had QTc prolongation at time of admission, and nearly $20 \%$ of those were admitted with a QTc greater than $500 \mathrm{~ms}$ [22]. A study showed the prevalence of prolonged QTc to be even higher in an acute geriatric ward [23]. Forty percent of hospitalized patients with a QTc greater than $500 \mathrm{~ms}$ have been observed to receive drugs associated with further prolongation of the QTc.

The most common agents that predispose to TdP are class I and III antiarrhythmics. These drugs are associated with TdP in more than $8 \%$ of cases [15]. Of the noncardiac drugs, it has been proposed that macrolides are the antibiotics with the greatest QTc prolonging potential [24]. This effect is multifactorial as macrolides not only block $\mathrm{I}_{\mathrm{k}}$ 
channels, but are also metabolized by CYP3A4, an isoenzyme responsible for the metabolism of over $60 \%$ of medications whose function is inhibited by many commonly prescribed drugs [25]. Macrolides are therefore more dangerous for those who simultaneously take CYP3A4 inhibitors or another QTc prolonging medication metabolized by this isoenzyme $[17,26]$. For example, use of clarithromycin alone increases the QTc by $6 \mathrm{~ms}$, but coadministration with cisapride, a prokinetic agent associated with QTc prolongation and also metabolized by CYP3A4, increases the QTc by $25 \mathrm{~ms}[27,28]$.

Azithromycin has been shown to have less effect on CYP3A4 and is more widely used than other macrolides [27, 29]. Between January 2002 and December 2011, azithromycin was mentioned $69,790,000$ times during outpatient office visits in connection with bronchitis. This represented $40.6 \%$ of all drugs cited for this diagnosis. During this time, azithromycin was second only to amoxicillin for treatment of sinusitis, and was mentioned 34,077,000 times [30]. In 2012, 40.3 million people were prescribed azithromycin [30]. Interestingly, the risk of sudden cardiac death observed with azithromycin occurred only within a typical 5-day course [5]. The rate of druginduced TdP varies by medication class. In a populationbased study of 605,127 people taking fluoroquinolone antibiotics, ventricular arrhythmias were detected in 180 patients and cardiac arrest occurred in 555 patients that had prior exposure to a quinolone. However, this study had an extensive immeasurable time bias between drug exposure and event rate and did not take into account the incidence of sustained ventricular tachycardia in patients with known prolonged QT who subsequently received the medication [31]. However, in our previous work of 1,004 patients evaluating the incidence of $\mathrm{TdP}$ in patients with prolonged QTc who subsequently received levofloxicin, $0.2 \%$ of patients experienced sustained ventricular tachycardia [32]. This is in contrast to our current study noting an event rate of $0.97 \%$. The rate of ventricular arrhythmia in our study was far too small to speculate about potential risk factors that contribute to the development of ventricular tachycardia in patients with prolonged QTc who are given azithromycin. However, consistent with the risk noted by Ray et al. [5], our lone case of ventricular tachycardia occurred during a 5-day course of therapy.

Our study included subjects with prolonged QTc, though we are uncertain if any patients within the cohort had a prior history of long QT syndrome. Although $43 \%$ of patients in this study were taking antidepressants, antifungals or antipsychotics, we did not account for a number of other commonly prescribed drug classes such as antiemetics and prokinetics that also prolong the QTc. To fully account for all medications that influence cardiac repolarization would be a monumental undertaking $[17,33]$.
Although the reasons why physicians in this study chose to use azithromycin in the presence of a prolonged QTc were not analyzed, the high degree of complexity and acuity of illness may have justified it. Furthermore, $58 \%$ of patients in the cohort were admitted with a primary diagnosis of respiratory illness and $66 \%$ of patients treated with azithromycin had pneumonia. Current guidelines for patients hospitalized with pneumonia recommend combination therapy with macrolides as first-line treatment [34] and emerging data suggest that azithromycin is associated with a reduction in 90-day mortality in patients with pneumonia [3]. However, this study was intended to provide new information on only the risk of sustained ventricular tachycardia in patients with prolonged QTc, not to partition the global risks and benefits of azithromycin which will vary by indication.

Our study has several limitations. First, the size of the study may not be large enough to detect the true incidence of antibiotic-instigated TdP. Second, the retrospective nature of the study may introduce bias as it relies on accurate charting. Third, the incidence of antibiotic-induced Tdp is difficult to estimate, and the arrhythmia is often self-limited, and thus may be missed. Two-thirds of cases of SCD or syncope have no record of a preceding arrhythmia [15]. However, a high percentage of our patients were monitored on telemetry, which may have aided in the identification of ventricular tachycardia. In addition, all QTc values were measured by computerbased analysis. Well-designed algorithms can accurately assess the QTc $95 \%$ of the time, and it has been suggested that the detection of drug-induced effects on ECG intervals is markedly enhanced by the use of computerbased analysis [35]. Fourth, patients with bundle branch blocks or implantable pacemakers were not analyzed independently. This may have led to an overestimation of subjects with prolonged QTc due to repolarization abnormalities. Fifth, as $91 \%$ of our cohort was Caucasian, the results of this study may not be generalizable to all populations. Finally, only short-term, in-hospital outcomes were observed. The long-term occurrence of sustained ventricular tachycardia following administration of azithromycin in patients with prolonged QT is unknown. However, prior studies noted the risk of cardiac death associated with azithromycin is present only during a course of treatment [5].

To our knowledge, this is the first study that examined the incidence of ventricular tachycardia in patients with prolonged QTc who were subsequently given azithromycin. Although there is no consensus on the degree of QTc prolongation that is clinically significant, caution should be exercised when prescribing medications that further prolong the QTc interval [36]. The Mayo Clinic has adopted an institution-wide QTc alert system to notify 
providers when a patient has prolonged QTc. This system has determined that a QTc greater than $500 \mathrm{~ms}$ is a strong predictor of all-cause, inpatient mortality, as seen in the one event in this cohort. In fact, mortality rates in patients with critically long QTc intervals are greater with increasing numbers of modifiable risk factors for TdP (electrolyte disturbances, polypharmacy) [37]. There is evidence that these risk factors contribute to greater mortality than do non-modifiable factors such as age and gender. Institutional alert systems may help providers mitigate modifiable risk factors, or minimize unnecessary drug-drug interactions [37, 38]. Other drugs that prolong the QTc require providers to monitor the patient for ECG changes. For example, the antiarrhythmic dofetilide should only be started in the hospital setting with serial monitoring of the QTc and electrolytes [39]. A similar strategy could be useful when prescribing other QTcprolonging medications in patients with a QTc greater than $500 \mathrm{~ms}$.

\section{Conclusion}

Based on the study results capturing patients treated with multiple doses of azithromycin in the setting of QTc prolongation over a 3-year period, we observed that the occurrence of sustained ventricular tachycardia in patients with prolonged QTc interval who subsequently received azithromycin may be less than $1 \%$. As azithromycin is widely prescribed and polypharmacy becomes more prevalent in the aging population, larger studies are needed to fully understand the implications of using macrolides in patients with prolonged QTc.

\section{Compliance with Ethical Standards}

Author contributions Steven Sears had full access to all of the data in the study and takes responsibility for the integrity of the data and the accuracy of the data analysis, including and especially any adverse effects. Steven Sears, Trevor Getz, Christopher Austin, William Palmer, Evelyn Boyd, and Fernando Stancampiano contributed substantially to the study design, data analysis and interpretation, and the writing of the manuscript.

Ethical approval The study was approved by the Mayo Clinic Institutional Review Board, study ID 14-005155. It has been performed in accordance with the ethical standards of the declaration of Helsinki.

Funding No sources of funding were used to assist in the preparation of this study.

Conflicts of interest Steven P. Sears, Trevor W. Getz, Christopher O. Austin, William C. Palmer, Evelyn A. Boyd, and Fernando F. Stancampiano have no conflicts of interest and nothing to disclose.
Open Access This article is distributed under the terms of the Creative Commons Attribution-NonCommercial 4.0 International License (http://creativecommons.org/licenses/by-nc/4.0/), which permits any noncommercial use, distribution, and reproduction in any medium, provided you give appropriate credit to the original author(s) and the source, provide a link to the Creative Commons license, and indicate if changes were made.

\section{References}

1. Ray WA, Murray KT, Meredith S, Narasimhulu SS, Hall K, Stein $\mathrm{CM}$. Oral erythromycin and the risk of sudden death from cardiac causes. N Engl J Med. 2004;351(11):1089-96.

2. Inserts P. Zithromax (azithromycin for oral and injection) Package Inserts 4763, 5179.5191.

3. Ray WA. Azithromycin associated with a reduction in 90-day mortality among older pneumonia patients, although a true clinical benefit is uncertain. Evid Based Med. 2014;19(6):226-7.

4. Poluzzi E, Raschi E, Moretti U, De Ponti F. Drug-induced torsades de pointes: data mining of the public version of the FDA Adverse Event Reporting System (AERS). Pharmacoepidemiol Drug Saf. 2009;18(6):512-8.

5. Ray WA, Murray KT, Hall K, Arbogast PG, Stein CM. Azithromycin and the risk of cardiovascular death. N Engl J Med. 2012;366(20):1881-90.

6. Rautaharju PM, Surawicz B, Gettes LS, Bailey JJ, Childers R, Deal BJ, et al. AHA/ACCF/HRS recommendations for the standardization and interpretation of the electrocardiogram: part IV: the ST segment, $\mathrm{T}$ and $\mathrm{U}$ waves, and the QT interval: a scientific statement from the American Heart Association Electrocardiography and Arrhythmias Committee, Council on Clinical Cardiology; the American College of Cardiology Foundation; and the Heart Rhythm Society. Endorsed by the International Society for Computerized Electrocardiology. J Am Coll Cardiol. 2009;53(11):982-91.

7. Eriksson JW, Carlberg B, Hillorn V. Life-threatening ventricular tachycardia due to liquorice-induced hypokalaemia. J Intern Med. 1999;245(3):307-10.

8. Elming H, Brendorp B, Kober L, Sahebzadah N, Torp-Petersen C. QTc interval in the assessment of cardiac risk. Card Electrophysiol Rev. 2002;6(3):289-94.

9. Rakic D, Rumboldt Z, Carevic V, Bagatin J, Polic S, Pivac N, et al. In-hospital cardiac arrest and resuscitation outcomes: rationale for sudden cardiac death approach. Croat Med J. 2005;46(6):907-12.

10. Myerburg RJ, Kessler KM, Castellanos A. Sudden cardiac death: epidemiology, transient risk, and intervention assessment. Ann Intern Med. 1993;119(12):1187-97.

11. Zipes DP, Wellens HJ. Sudden cardiac death. Circulation. 1998;98(21):2334-51.

12. Zheng ZJ, Croft JB, Giles WH, Mensah GA. Sudden cardiac death in the United States, 1989 to 1998. Circulation. 2001;104(18):2158-63.

13. Pickham D, Helfenbein E, Shinn JA, Chan G, Funk M, Weinacker A, et al. High prevalence of corrected QT interval prolongation in acutely ill patients is associated with mortality: results of the QT in Practice (QTIP) Study. Crit Care Med. 2012;40(2):394-9.

14. Anderson KP. The changing epidemiology of ventricular arrhythmias. Cardiol Clin. 2008;26(3):321-33 (v).

15. Shaffer D, Singer S, Korvick J, Honig P. Concomitant risk factors in reports of torsades de pointes associated with macrolide use: 
review of the United States Food and Drug Administration Adverse Event Reporting System. Clin Infect Dis. 2002;35(2):197-200.

16. Beitland S, Platou ES, Sunde K. Drug-induced long QT syndrome and fatal arrhythmias in the intensive care unit. Acta Anaesthesiol Scand. 2014;58(3):266-72.

17. Gupta A, Lawrence AT, Krishnan K, Kavinsky CJ, Trohman RG. Current concepts in the mechanisms and management of druginduced QT prolongation and torsade de pointes. Am Heart J. 2007;153(6):891-9.

18. Owens RC Jr. QT prolongation with antimicrobial agents: understanding the significance. Drugs. 2004;64(10):1091-124.

19. Haverkamp W, Breithardt G, Camm AJ, Janse MJ, Rosen MR, Antzelevitch $\mathrm{C}$, et al. The potential for QT prolongation and proarrhythmia by non-antiarrhythmic drugs: clinical and regulatory implications. Report on a policy conference of the European Society of Cardiology. Eur Heart J. 2000;21(15):1216-31.

20. Al-Khatib SM, LaPointe NM, Kramer JM, Califf RM. What clinicians should know about the QT interval. JAMA. 2003;289(16):2120-7.

21. Armahizer MJ, Seybert AL, Smithburger PL, Kane-Gill SL. Drug-drug interactions contributing to QT prolongation in cardiac intensive care units. J Crit Care. 2013;28(3):243-9.

22. Tisdale JE, Wroblewski HA, Overholser BR, Kingery JR, Trujillo TN, Kovacs RJ. Prevalence of QT interval prolongation in patients admitted to cardiac care units and frequency of subsequent administration of QT interval-prolonging drugs: a prospective, observational study in a large urban academic medical center in the US. Drug Saf. 2012;35(6):459-70.

23. Lubart E, Segal R, Yearovoi A, Fridenson A, Baumoehl Y, Leibovitz A. QT interval disturbances in hospitalized elderly patients. Isr Med Assoc J. 2009;11(3):147-50.

24. Owens RC Jr. Risk assessment for antimicrobial agent-induced QTc interval prolongation and torsades de pointes. Pharmacotherapy. 2001;21(3):301-19.

25. Dresser GK, Spence JD, Bailey DG. Pharmacokinetic-pharmacodynamic consequences and clinical relevance of cytochrome P450 3A4 inhibition. Clin Pharmacokinet. 2000;38(1):41-57.

26. Stanat SJ, Carlton CG, Crumb WJ Jr, Agrawal KC, Clarkson CW. Characterization of the inhibitory effects of erythromycin and clarithromycin on the HERG potassium channel. Mol Cell Biochem. 2003;254(1-2):1-7.

27. Owens RC Jr, Nolin TD. Antimicrobial-associated QT interval prolongation: pointes of interest. Clin Infect Dis. 2006;43(12): 1603-11.
28. Desta Z, Kerbusch T, Flockhart DA. Effect of clarithromycin on the pharmacokinetics and pharmacodynamics of pimozide in healthy poor and extensive metabolizers of cytochrome P450 2D6 (CYP2D6). Clin Pharmacol Ther. 1999;65(1):10-20.

29. Harris S, Hilligoss DM, Colangelo PM, Eller M, Okerholm R. Azithromycin and terfenadine: lack of drug interaction. Clin Pharmacol Ther. 1995;58(3):310-5.

30. Mosholder AD, Mathew J, Alexander JJ, Smith H, Nambiar S. Cardiovascular risks with azithromycin and other antibacterial drugs. N Engl J Med. 2013;368(18):1665-8.

31. Lapi F, Wilchesky M, Kezouh A, Benisty JI, Ernst P, Suissa S. Fluoroquinolones and the risk of serious arrhythmia: a population-based study. Clin Infect Dis. 2012;55(11):1457-65.

32. Stancampiano FF, Palmer WC, Getz TW, Serra-Valentin NA, Sears SP, Seeger KM, et al. Rare Incidence of Ventricular Tachycardia and Torsades de Pointes in Hospitalized Patients With Prolonged QT Who Later Received Levofloxacin: a Retrospective Study. Mayo Clin Proc. 2015;90(5):606-12.

33. Kallergis EM, Goudis CA, Simantirakis EN, Kochiadakis GE, Vardas PE. Mechanisms, risk factors, and management of acquired long QT syndrome: a comprehensive review. Sci World J. 2012;2012:212178.

34. Mandell LA, Wunderink RG, Anzueto A, Bartlett JG, Campbell GD, Dean NC, et al. Infectious Diseases Society of America/ American Thoracic Society consensus guidelines on the management of community-acquired pneumonia in adults. Clin Infect Dis. 2007;1(44 Suppl 2):S27-72.

35. Guth BD, Bass AS, Briscoe R, Chivers S, Markert M, Siegl PK, et al. Comparison of electrocardiographic analysis for risk of QT interval prolongation using safety pharmacology and toxicological studies. J Pharmacol Toxicol Methods. 2009;60(2):107-16.

36. Malik M, Camm AJ. Evaluation of drug-induced QT interval prolongation: implications for drug approval and labelling. Drug Saf. 2001;24(5):323-51.

37. Haugaa KH, Bos JM, Tarrell RF, Morlan BW, Caraballo PJ, Ackerman MJ. Institution-wide QT alert system identifies patients with a high risk of mortality. Mayo Clin Proc. 2013;88(4):315-25.

38. Giudicessi JR, Ackerman MJ. Azithromycin and risk of sudden cardiac death: guilty as charged or falsely accused? Cleve Clin J Med. 2013;80(9):539-44.

39. Friedman L, Alexander E. Update of the clinical impact and issues surrounding Dofetilide (Tikosyn) therapy. AACN Adv Crit Care. 2006;17(2):102-8 (quiz 9-10). 